\title{
MODELING THE DIVERSE FUV SPECTRA OF DISK-DOMINATED CATACLYSMIC VARIABLES
}

\author{
Knox S. Long' and ('luristian Knigge ${ }^{2}$
}

\section{RESUTIEN}

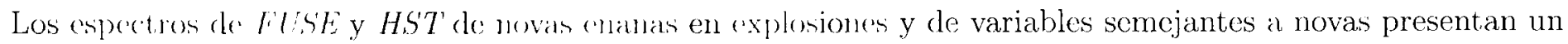
amplio rango le aracterísticas. En unos claantos sistrmas existen señales obvias de un viento rápido en la forma de los perfiles do ()VI somejantess a P- ('ygni y de otros iones en relativamente altos estados de ionización. Pero (atos casos son raros. Son más comuncs los sistomat con espectros FUV que presentan una compleja mezcla de líneas de allo y hajo astado de ionización. Su describen intentos para reproducir los espectros utilizando el ádige dr Monte ('arlo de transferenciat rarliativa, desarrollado para modelar vientos bicónicos en sistemas de disco.

\section{ABSTRACT}

The FlSE and HST spectra of dwarf nove in outburst and of nova-like variables exhibit a wide range of characteristics. In a few systems, there are obvious signatures of a fast wind in the form of P-Cygni-like profiles of OVI and other matively high ionization state ions. But this is rare. More common are systems with liv spertri showing a complex mixture of high and low ionization state lincs. Here we describe attempts to reproduce the spectra using a Monte ('arlo radiative transfer code developed to model bi-conical winds in disk sistoms.

\section{Key Words: ACCRETION, ACCRETION DISKS - BINARIES: CLOSE - NOVAE, CATACLYSMIC VARIABLES - STARS: MASS LOSS}

Since the original /lW studies of catalysmie variables. it has bern rlat that nova-like variables and dwarf nowa in outbursts drive winds (Heap et al.

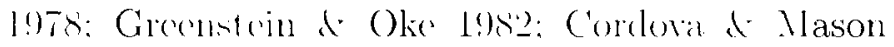
195:). The cridene for winds is found in P'-('ymnilike profiles of ('Il' and other strong resonanee lines in many systents, and in bluc-shifted entromids of the same liness in many others. The blue-edge velecition in systents. such ats IX Vol and V3885 Sgr (Hartley of al. $2(012)$ are $2000(-5000) \mathrm{km} s \mathrm{~s}^{1}$. In high-inclination sistems. the resonane lines are seen primarily in enission. Time-resolved studies of the line shapess show rotational effects (Mason of al. 1995). leading to qualitative picture of the wind in terms a last bipolar flow originating from the inner disk. A good description of the wind has been sery difficult to develop. Furthermore, many the disk-dominated cataclysmic rariables observed with FLSE show lines with arising from low velocity: relatively low ionization state transitions,prestunably arising from material further out from in the disk whose origin is still poorly understood (Sore e.g. Froning et al. 2001).

In an attenpt to develop a better understanding of the structure of the out flow that develops around

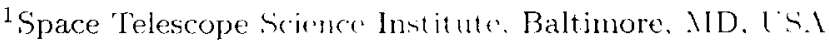

${ }^{2} \mathrm{E}$. of Southampton. $1 \mathrm{~h}$
}

the disk of nova-like variables and of dwarf novae in outburst, we have developed a Mont "Carlo radiative transfer code to simulate the UV and FUV spectra assuming an axially symmetric wind flow (Long d Kinigge 2002). Unlike Shlosman \& Vitello (1993) and Knigge et al.,(1995). who concentrated solely on modeling individual lines, usually C IV, our goal is to simulate the entire spectrum. The code, Python. invokes a Sobolev approximation with escape probabilities to follow photons through the wind. We do not attempt to calculate self-consistently the flow, by for example, calculating the radiative pressure, and modifying the flow. Instead, the wind geometry and flow are defined using the prescriptions of Shlosman \& Vitello (1993) or Knigge et al., (1995). All cosmically-abundant elements are included. Sources of radiation include the disk, the white dwarf, the boundary layer (modeled as an additional source lo(ated at the white dwarf). and the wind itself.

Simulated spectra generated with Python resemble those observed with FUSE, HST, and other space-borne observatories. Nost of the transitions that are commonly seen in CVs are seen in the models witl about the correct line strengths. In addition, the simulated spectra also indicate all of the basic characteristics that one would cxpect as system pa- 

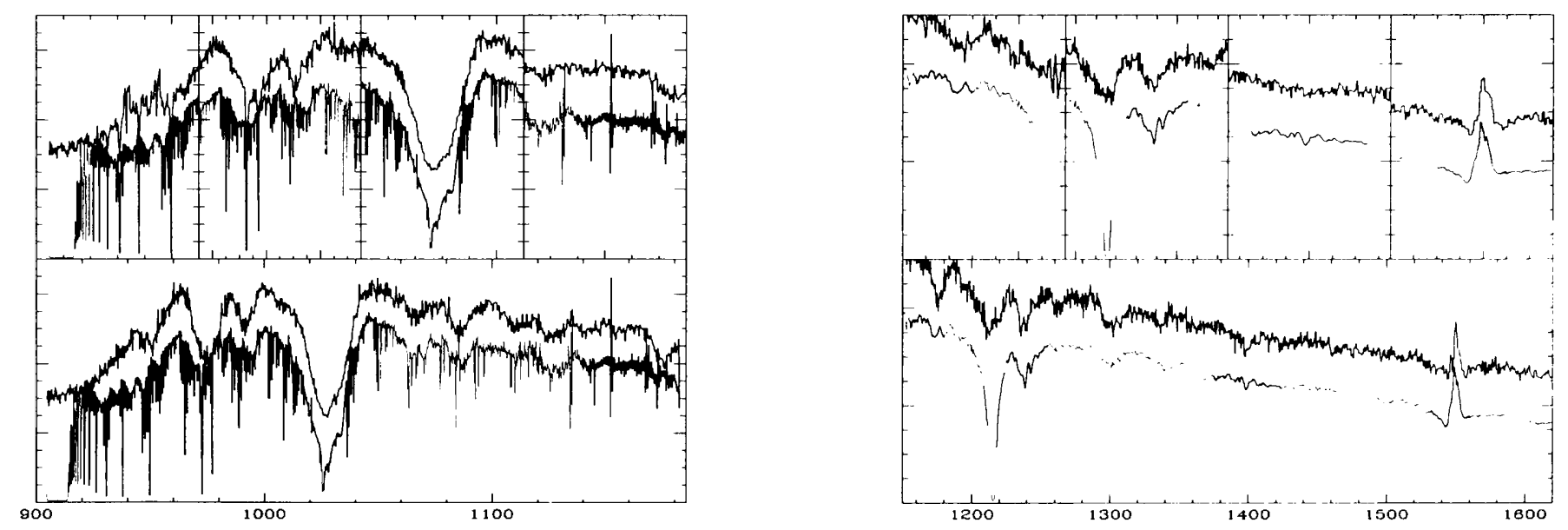

Fig. 1. Example of a fit to the FUSE (left) and HST/GHRS (right) spectra of SS Cygni in outburst. The upper panels show fits to individual regions of the spectra. The lower panels are consolidated fits to the FlSF and HST'spectra. For clarity, the models are shown displaced above the data. The best-fit models in the two cats are similar. Inu not identical.

rameters are changed: a transition from pure absorption to pure emission as the inclination is increased, a progression towards higher ionization state lines as $\dot{m}_{d i s k}$ is increased or $\dot{m}_{w i n d}$ is decreased, and the proper rotational effects in line profiles as eclipses are simulated.

We are currently beginning an effort to construct large grids of models in an attempt to determine to what degree the wind prescriptions built into the code can reproduce the spectra of specific CVs in a quantitative sense. As one would expect, it is relatively easy to fit individual lines, especially the strong resonance lines.

Our most encouraging results are obtained on lower inclination systems. For example, as shown in Fig. 1, we are able to fit both FUSE (900-1185 $\AA$ ) and HST/GHRS (1150-1640 $\AA$ ) spectra fairly well with a single wind model for each. The wind prescriptions that fit best at present require $\dot{m}_{\text {wind }}$ or 0.01-0.1 $\dot{m}_{\text {disk }}$ with a hint that the lower value is closer to the norm. They also favor relatively long acceleration lengths, as was found by Shlosman et al., (1996) and by Knigge \& Drew(1997) in their attempts to model C IV in a number of CVs.

Others systems, particularly, those which are observed at high inclination, such as WZ Sge and UX UMa, are more problematic. This is in part because it is very difficult to fit both the high ionization lines that participate in the fast outflow and the low ionization lines that do not.

However, it is not yet evident that we have explored all of the relevant portions of parameter space, and so it is not yet clear how well we will do in the end. Ideally, we will find that a group of models in a restricted range of parameters fit the spectra of a variety of disks and winds. This would allow one to place real physical limits on the nature of the winds in disk-dominated cataclysmic variables.

This work was supported by NASA through grants associated with our analysis of HST and FUSE spectra of dwarf novae. Specifically we acknowledge support from grants GO-8279 from the Space Telescope Science Institute, which is operated by AURA, Inc., under NASA contract NAS5-2(5.5.5. as well from grants NAG5-10338 and NA(i.)-11 Nit.). which are associated with our FUSE projects.

\section{REFERENCES}

Cordova, F. A. \& Mason, K. O. 19\&2, ApJ, 260, 716

Froning, C. S., Long, K. S., Drew, J. E.. Kininge. ( .. \& Proga, D. 2001, ApJ, 562, 96:3

Greenstein, J. L. \& Oke, J. B. 1982. Ap.J, 258. 2019

Hartley, L. E., Drew, J. E., Long, K. S., Knigge. C.. \& Proga, D. 2002, MNRAS, 332, 127

Heap, S. R. et al. 1978, Nature. 275. 385

Knigge, ('. Woods. J. A., \& Drew, E. 1995\%, MNR: L 273,225

Knigge, ('. \& Drew, J. E. 1997. ApJ, hist 4t)

Long, K. S. \& Knigge. ( .2002 , АpJ , , 79.725

Mason, K. O., Drew, J. F.. Cordova, F. A. Horne. h.. Hilditch, R., Knigge, ( . Lamz. T. d Merlan. T 196). MNRAS, $27 \cdot 1,271$

Shlosman, I. d Vitello, P 19993. Apro, 109, :37-2

Shlosman. I. Vitcllo. P.. \& Mauche, C. II 1!)(i. Mr.l. 161,377 